\title{
Co-evolving wing spots and mating displays are genetically separable traits in Drosophila
}

\author{
Jonathan H. Massey, ${ }^{1,2}$ iD Gavin R. Rice, ${ }^{3}$ iD Anggun S. Firdaus, ${ }^{4}$ Chi-Yang Chen, ${ }^{4}$ Shu-Dan Yeh, ${ }^{4}$ iD \\ David L. Stern, 2,5 iD and Patricia J. Wittkopp ${ }^{1,6,7}$ iD \\ ${ }^{1}$ Department of Ecology and Evolutionary Biology, University of Michigan, Ann Arbor, Michigan 48109 \\ 2 Janelia Research Campus, Howard Hughes Medical Institute, Ashburn, Virginia 20147 \\ ${ }^{3}$ Department of Biological Sciences, University of Pittsburgh, Pittsburgh, Pennsylvania 15260 \\ ${ }^{4}$ Department of Life Sciences, National Central University, Taoyuan 32001, Taiwan \\ ${ }^{5}$ E-mail: sternd@janelia.hhmi.org \\ ${ }^{6}$ Department of Molecular, Cellular, and Developmental Biology, University of Michigan, Ann Arbor, Michigan 48109 \\ ${ }^{7}$ E-mail: wittkopp@umich.edu
}

Received December 11, 2019

Accepted April 27, 2020

\begin{abstract}
The evolution of sexual traits often involves correlated changes in morphology and behavior. For example, in Drosophila, divergent mating displays are often accompanied by divergent pigment patterns. To better understand how such traits co-evolve, we investigated the genetic basis of correlated divergence in wing pigmentation and mating display between the sibling species Drosophila elegans and Drosophila gunungcola. Drosophila elegans males have an area of black pigment on their wings known as a wing spot and appear to display this spot to females by extending their wings laterally during courtship. By contrast, $D$. gunungcola lost both of these traits. Using Multiplexed Shotgun Genotyping (MSG), we identified a $\sim 40$ kb region on the X chromosome that behaves like a genetic switch controlling the presence or absence of male-specific wing spots. This region includes the candidate gene optomotor-blind (omb), which plays a critical role in patterning the Drosophila wing. The genetic basis of divergent wing display is more complex, with at least two loci on the $X$ chromosome and two loci on autosomes contributing to its evolution. Introgressing the X-linked region affecting wing spot development from $D$. gunungcola into $D$. elegans reduced pigmentation in the wing spots but did not affect the wing display, indicating that these are genetically separable traits. Consistent with this observation, broader sampling of wild $D$. gunungcola populations confirmed that the wing spot and wing display are evolving independently: some $D$. gunungcola males performed wing displays similar to $D$. elegans despite lacking wing spots. These data suggest that correlated selection pressures rather than physical linkage or pleiotropy are responsible for the coevolution of these morphological and behavioral traits. They also suggest that the change in morphology evolved prior to the change in behavior.
\end{abstract}

KEY WORDS: Correlated traits, courtship behavior, Drosophila, optomotor-blind, pigmentation.

Animals often use colorful morphological structures to communicate with prospective mates during courtship (McKinnon and Pierotti 2010). In vertebrates and invertebrates, pigmented bodies or wings often evolve together with specific components of courtship behavior that animals use to display their colorful anatomy (Loxton 1979; Endler 1991; Sinervo et al. 2000; White et al. 2015). These correlated differences evolve both within and between populations, frequently distinguishing males from females or closely related species (Gray and McKinnon 2007; McKinnon and Pierotti 2010). In the handful of case studies examining the genetic basis of such co-evolving traits, linkage mapping and genome-wide association studies (GWAS) have shown that loci affecting pigmentation patterning tend to map to the same region of the genome as loci affecting variation in 
mating behaviors (Lindholm and Breden 2002; Kronforst et al. 2006; Yeh et al. 2006; Thomas et al. 2008; Küpper et al. 2016; Lamichhaney et al. 2016; Merrill et al. 2019; reviewed in McKinnon and Pierotti 2010). That is, physical linkage of genetic variants often appears to underlie phenotypic correlations between mating behavior and pigmentation. Although linked loci tend to explain much of the variation observed for both traits, it remains unclear in all of these cases which genes underlie the observed correlations in phenotypic differences. A key challenge, therefore, is determining how frequently these patterns of genomic architecture underlie correlated evolution and whether a single pleiotropic locus or separate linked loci are involved.

Disentangling whether pleiotropic or physically linked loci underlie patterns of correlated evolution between pigmentation and mating behavior is important for understanding how natural selection generates differences between sexes and species. If two beneficial traits are genetically correlated due to separate, physically linked loci, theory predicts that natural or sexual selection (e.g., through predation or female choice) will act to minimize recombination between the causal loci (Charlesworth and Charlesworth 1976). It has been hypothesized that one solution to this problem might involve the evolution of chromosomal inversions that suppress recombination between two or more linked loci (Kirkpatrick and Barton 2006), and in multiple cases linked loci contributing to correlated evolution have been shown to lie within inversions (Thomas et al. 2008; Küpper et al. 2016; Lamichhaney et al. 2016). Alternatively, mutations at a single pleiotropic gene could cause correlated components of pigmentation and mating behavior to evolve simultaneously. Although such mutations have not yet been identified for pigmentation and behavior, they have been found for other co-evolving traits (e.g., Chang et al. 2009; Kent et al. 2009; McLean et al. 2011; Duveau and Félix, 2012; Endler et al. 2018; Nagy et al. 2018). Distinguishing between these genetic modes of phenotypic evolution requires, in part, high-resolution mapping of correlated traits.

In the Oriental Drosophila melanogaster species group, male-specific wing spots are phylogenetically correlated with mating displays (Kopp and True 2002; Fig. 1A). Species with wing spots perform elaborate wing display dances during courtship, extending their wings laterally, turning their dorsal wing surfaces toward the female, and waving them up and down; species without wing spots lack display behavior (Kopp and True 2002; Fig. 1A,B). Correlated gains and losses of both traits have evolved repeatedly (Kopp and True 2002; Fig. 1A). For example, in D. elegans and D. gunungcola, sibling species from this group that are estimated to have diverged 2-2.8 million years ago (Prud'homme et al. 2006), D. elegans (Bock and Wheeler 1972) males possess wing spots and perform wing displays, whereas $D$. gunungcola (Sultana et al. 1999) males lost both traits (Kopp and True 2002; Prud'homme et al. 2006; Yeh et al. 2006; Fig. 1B;
Videos 1 and 2). Previously, Yeh et al. (2006) and Yeh and True (2014) discovered that D. elegans and D. gunungcola can generate fertile $F_{1}$ hybrid female offspring in the lab and they performed interspecific crosses to study the genetic basis of wing spot and wing display divergence. Through quantitative trait locus (QTL) mapping, they showed that evolution of linked loci on the $\mathrm{X}$ chromosome contributed to divergence in both traits (Yeh et al. 2006; Yeh and True 2014). One QTL explaining wing spot size variation was linked to the pigmentation gene yellow, supporting the hypothesis that yellow cis-regulatory divergence contributes to wing pigmentation evolution (Wittkopp et al. 2002a; Gompel et al. 2005; Prud'homme et al. 2006). It remained unclear, however, whether the same or different loci on the $\mathrm{X}$ chromosome underlie correlated differences in wing spot and wing display between these species.

To distinguish between these possibilities, we re-examined the genetic basis of wing spots and wing display divergence between D. elegans and D. gunungcola. Specifically, we (1) generated recombinant backcross progeny segregating for both traits, (2) assembled chromosome-length scaffolds of D. elegans, (3) used Multiplexed Shotgun Genotyping (MSG) (Andolfatto et al. 2011) to estimate recombination crossover positions across the genome, (4) generated quantitative measures of both wing spots and wing display behavior to estimate the effect size of loci contributing to divergence, and (5) generated advanced, recombinant introgressions on the $\mathrm{X}$ chromosome in an attempt to separate quantitative trait loci (QTL) underlying wing spots and wing display behavior. These experiments showed that a single locus on the $\mathrm{X}$ chromosome behaves like a genetic switch for wing spot divergence. Males with their wing spots turned off via introgression, however, performed wing displays like D. elegans males, indicating that the two traits are genetically separable. These findings suggest that wing spot and wing display behavior might have originally diverged independently. Consistent with this hypothesis, newly collected D. gunungcola strains from Indonesia appear to completely lack wing spots but retain the ability to perform wing displays. This observation suggests that the loss of wing spots occurred prior to the loss of wing display in the reference strain of D. gunungcola used in this study and in prior work.

\section{Materials and Methods FLY STOCKS}

The D. elegans HK (Hong Kong) and D. gunungcola SK (Sukarami) lines used in this study were a gift from John True (Stony Brook University). Species stocks were kept on a $12 \mathrm{~h}$ light-dark cycle at $23^{\circ} \mathrm{C}$ on a University of Michigan " $\mathrm{R}$ food" diet containing molasses (http://labexpress.com/flyfoodsupplies.htm\#rfood) (Wirtz and Semey 

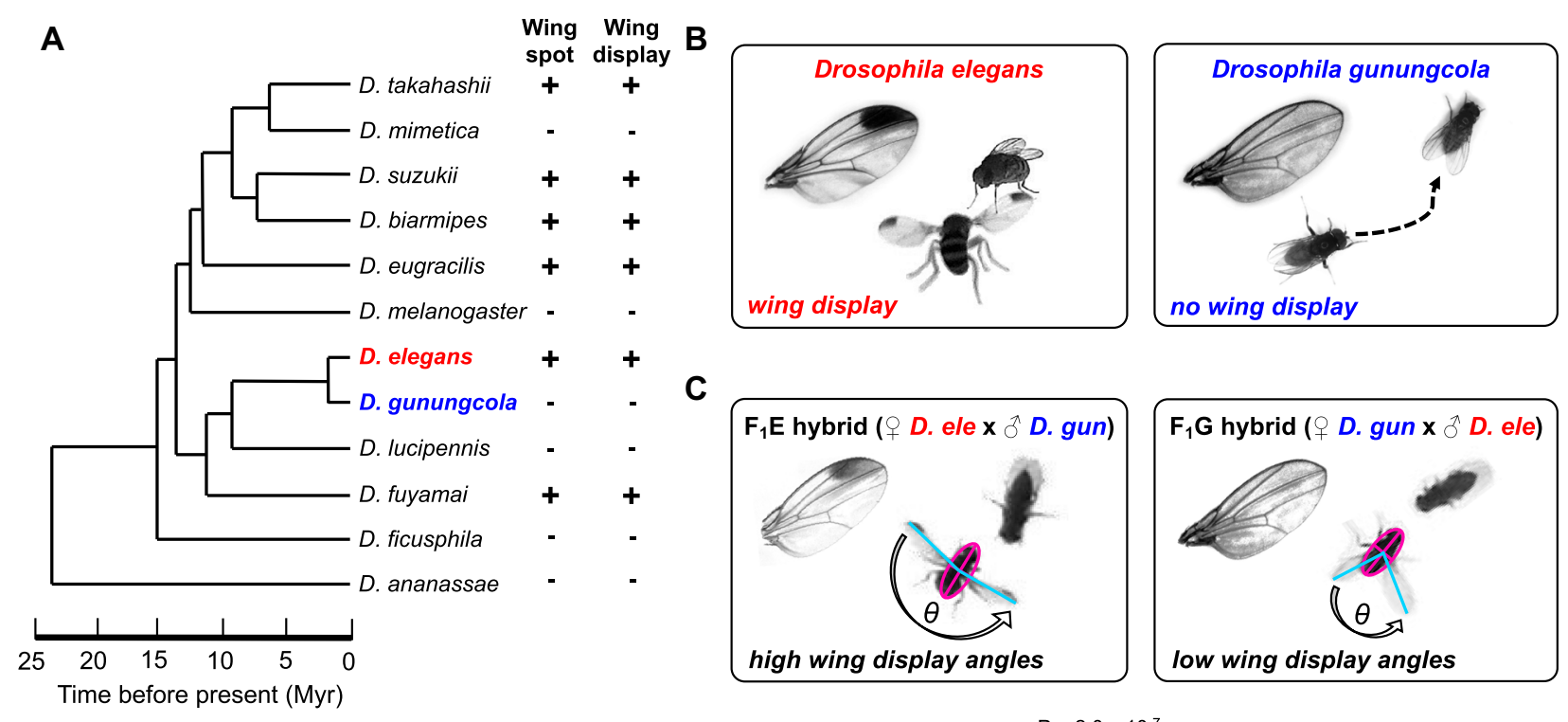

C
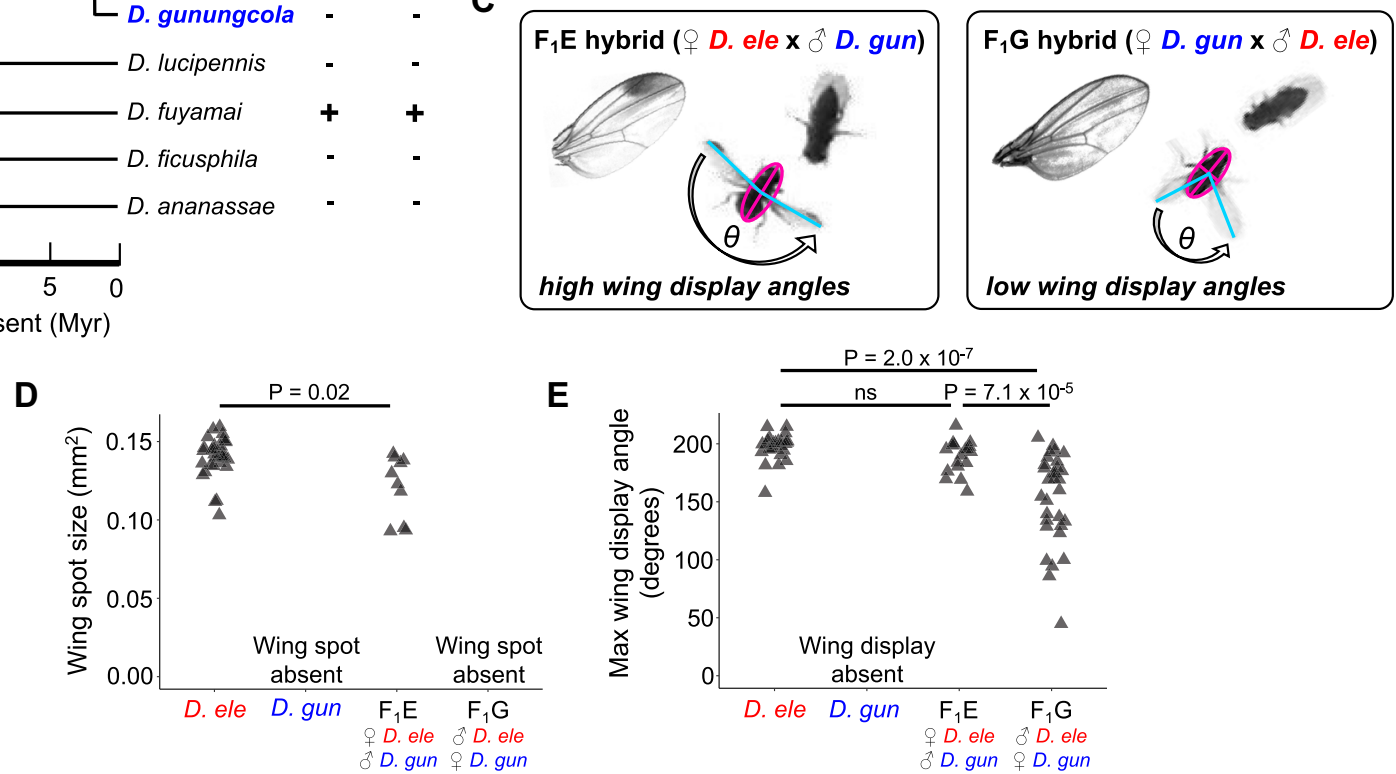

Figure 1. Wing pigmentation and wing display behavior in D. elegans, D. gunungcola, and $F_{1}$ hybrids. (A) Phylogeny of the "Oriental" Drosophila melanogaster species group adapted from Kopp and True (2002) and Prud'homme et al. (2006). Plus (+) signs indicate species possess wing spots and/or wing displays, and minus (-) signs indicates wing spots and/or wing displays are absent. (B) Males in $D$. elegans (left) possess wing spots and perform bilateral wing display behaviors in front of females during courtship (Video 1). Wing spots and wing displays are absent in $D$. gunungcola males (right) (Video 2). (C) $F_{1}$ hybrid males inheriting their $X$ chromosome from $D$. elegans mothers $\left(F_{1} E\right.$, left) possess wing spots and perform wing display behavior like $D$. elegans (Video 3 ). $F_{1}$ hybrid males inheriting their $X$ chromosome from $D$. gunungcola mothers $\left(F_{1} G\right.$, right) are spotless and perform wing displays with low bilateral wing angles (Video 4$)$. (D) Quantification of wing spot size (see Methods) in male $D$. elegans and $F_{1} E$. Wing spots are larger in $D$. elegans than $F_{1} E$ (Student's $t$-test; $t=-2.8057 ; d f=11.43 ; P=0.017$; two-tailed). (E) Quantification of maximum bilateral wing display angles during courtship (see Methods) in male $D$. elegans and $F_{1}$ hybrids. $F_{1} G$ hybrids showed lower maximum wing display angles than $D$. elegans and $F_{1} E$ hybrids (one-way ANOVA: $F_{2,71}=20.92 ; P<7.18 \times 10^{-8}$; post hoc Tukey HSD was significant between $D$. elegans and $F_{1} G$ : $P<2.0 \times 10^{-7}$ and between $F_{1} E$ and $F_{1} G: P<7.1 \times 10^{-5}$ ). Gray triangles represent individual replicates.

1982). Maintaining these species on $\mathrm{R}$ food at high densities (50100 flies per vial) allowed for the parental population to build up to thousands of flies to collect hundreds of virgins for interspecific crosses (see below). Neither D. elegans nor D. gunungcola pupate on the sides of the vial, so adults were flipped out when third instar L3 larvae developed and Fisherbrand filter paper (cat\# 09-790-2A) was added to the food to create pupation space.

\section{GENERATING HYBRID PROGENY}

Virgin males and females of D. elegans $H K$ and D. gunungcola $S K$ (the same lines used previously in Yeh et al. 2006; Yeh and True 2014) were isolated upon eclosion and stored in groups of 10 for one week on University of Michigan "M food," which is the standard cornmeal diet from the Bloomington Drosophila Stock Center (https://bdsc.indiana.edu/information/recipes/bloomfood.html) with $20 \%$ higher agar content. Virgin males from D. elegans were crossed to virgin females from D. gunungcola, and virgin males from D. gunungcola were crossed to virgin females from D. elegans in groups of 10 males and 10 females to generate fertile $F_{1}$ female and sterile $F_{1}$ male hybrids. These crosses took $\sim 3-4$ weeks to produce hybrid progeny. The switch from $\mathrm{R}$ food to $\mathrm{M}$ food for interspecific crosses was necessary, because $\mathrm{R}$ food tended to accumulate condensation 
and bacterial growth much faster than $\mathrm{M}$ food when few flies occupied a vial. Because crossing D. elegans and D. gunungcola to generate $F_{1}$ hybrids tends to take several more weeks than within species crosses, the switch to $M$ food diet allowed for maximum breeding time and the development of dozens of hybrid progeny. Once hybrid females eclosed from both interspecific cross directions, they were pooled into the same vial and aged for 10 days. We did not keep track of $F_{1}$ hybrid female maternity, because previous work (Yeh and True 2014) found no effect of $F_{1}$ hybrid maternity on trait means for wing spots and wing display in backcross populations. Multiple high-density groups of $\sim 60$ $\mathrm{F}_{1}$ hybrid females were then backcrossed to $\sim 60$ virgin male $D$. elegans flies in individual vials on $\mathrm{M}$ food diet to create the $D$. elegans backcross recombinant population (724 individuals). To create the D. gunungcola backcross recombinant population (241 individuals), groups of $\sim 60 \mathrm{~F}_{1}$ hybrid females were backcrossed to $\sim 60$ virgin male $D$. gunungcola flies in individual vials on $\mathrm{M}$ food diet; this backcross was less successful at producing recombinant progeny than the $D$. elegans backcross direction.

\section{BEHAVIORAL ASSAYS}

Virgin D. elegans females were isolated upon eclosion, aged 10-20 days, and stored in groups of 30-40 for courtship assays. $\mathrm{F}_{1}$ hybrid and recombinant backcross males were isolated individually in $\mathrm{M}$ food vials using $\mathrm{CO}_{2}$ upon eclosion for at least 5 days before each courtship assay. For each assay, a single individual male was gently aspirated into a custom built $70-\mathrm{mm}$ diameter bowl arena that matches the specifications in Simon and Dickinson (2010). Next, a single virgin D. elegans female was aspirated into the chamber and videotaped for the next 20 min, using a Canon VIXIA HF R500 camcorder mounted to Manfrotto (MKCOMPACTACN-BK) aluminum tripods. Videos were recorded between $0900 \mathrm{~h}$ and $1600 \mathrm{~h}$ at $23^{\circ} \mathrm{C}$. Drosophila elegans virgin females were used in all courtship assays in case any D. elegans female cues were necessary to elicit male wing display behavior. After each assay, both the male and female were aspirated back into an $\mathrm{M}$ food vial and left for up to 5 days, after which each male was frozen in individual $1.5 \mathrm{~mL}$ Eppendorf tubes for wing spot quantification (see MethodsQUANTIFICATION OF WING SPOTS), genomic DNA (gDNA) extraction, and sequencing (see Methods-LIBRARY PREPARATION AND SEQUENCING). All courtship videos ( $~ 900$ total) are available here: https://deepblue.lib.umich.edu/ data/concern/data_sets/j098zb17n?locale=en.

\section{QUANTIFICATION OF WING DISPLAY BEHAVIOR}

$F_{1}$ hybrid and recombinant males from both backcross directions performed variable wing display behaviors during courtship as described previously (Yeh et al. 2006; Yeh and True 2014). To generate quantitative measurements of wing display variation between individuals, each courtship video was played using QuickTime (version 10.4) (Apple Inc., Cupertino, CA) software in a MacOS environment, and digital screenshots were manually taken for each wing display bout, defined as a bilateral wing extension performed near the female (Fig. S1). Next, for each individual fly, wing display screenshots were compared to each other to identify the maximum wing display bout per fly, defined by comparing the distance between the tips of each wing relative to the center of the fly. These maximum wing display screenshots were then imported into ImageJ software (version 1.50i) (Wayne Rasband, National Institutes of Health, USA; http://rsbweb.nih.gov/ij/) to manually measure the "Maximum wing display angle" for $\mathrm{F}_{1}$ hybrid and recombinant males. In ImageJ, each screenshot image was inverted using the "Find Edges" function to enhance the contrast between the arena background and the edges of the fly wings (Fig. S1). Next, the "Polygon Selections" tool was used to fit an ellipse around the fly body using the "Fit Ellipse" function (Fig. S1). A Macros function (File S1) was then used to generate major and minor axes inside the ellipse to identify the center of the fly body (Fig. S1). Finally, the "Angle Tool" was used to measure the "Maximum wing display angle" centering the vertex at the intersection of the major and minor axes and extended from wing tip to wing tip (Fig. S1). "Maximum wing display angle" varied between $\sim 50^{\circ}$ and $\sim 220^{\circ}$ between backcross recombinant individuals. Raw data for Figure 1E are available in File S2, and raw data for Figure 3 are deposited on Dryad (https://doi.org/10.5061/dryad.gb5mkkwm5).

\section{QUANTIFICATION OF WING SPOTS}

Because wing spots fully form $\sim 24 \mathrm{~h}$ after eclosion in D. elegans, all parental male D. elegans, D. gunungcola, $\mathrm{F}_{1}$ hybrids, and backcross recombinants were aged at least 7 days before being frozen at $-20^{\circ} \mathrm{C}$ in $1.5 \mathrm{~mL}$ Eppendorf tubes. Next, using a 20 Gauge stainless steel syringe tip (Techcon) (cat\# TE720100PK), the right wing of each fly was cut away from the thorax and placed on a glass microscope slide (Fisherbrand) (cat\# 12-55015 ) to image using either a Leica MZFLIII stereoscope equipped with a Leica DC480 microscope camera or a Canon EOS Rebel T6 camera equipped with a Canon MP-E $65 \mathrm{~mm}$ macro lens. Each camera was calibrated using an OMAX 0.1-mm slide micrometer to define pixel density in ImageJ software. JPEG images of wings were imported into ImageJ to measure wing spot size relative to total wing area (wing spot size/total wing area). We quantified wing spot size, rather than wing spot intensity, because we aimed to map previously identified wing spot size QTL (Yeh et al. 2006; Yeh and True 2014) and their relationship with wing display behavior. Total wing area (wing length $\times$ wing width) was approximated using length and width proxies following methods described in Yeh and True (2014). Using the "Polygon Selections" tool, the margins of black pigmentation 
A

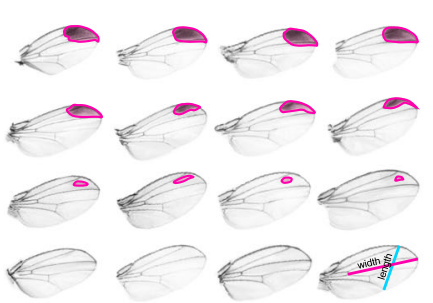

B

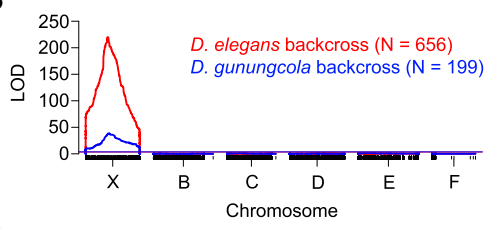

C

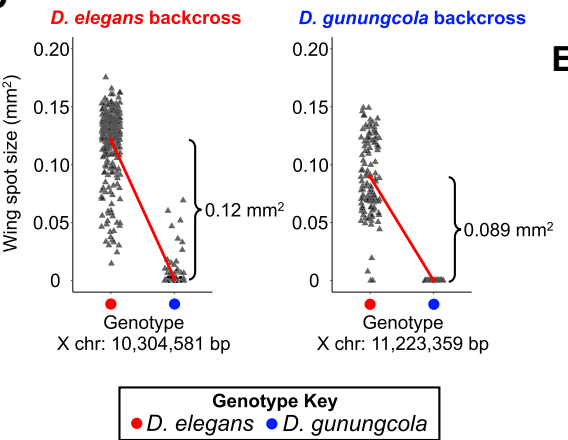

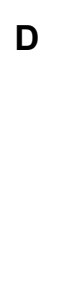

$\mathrm{X}$ chr.

20

E

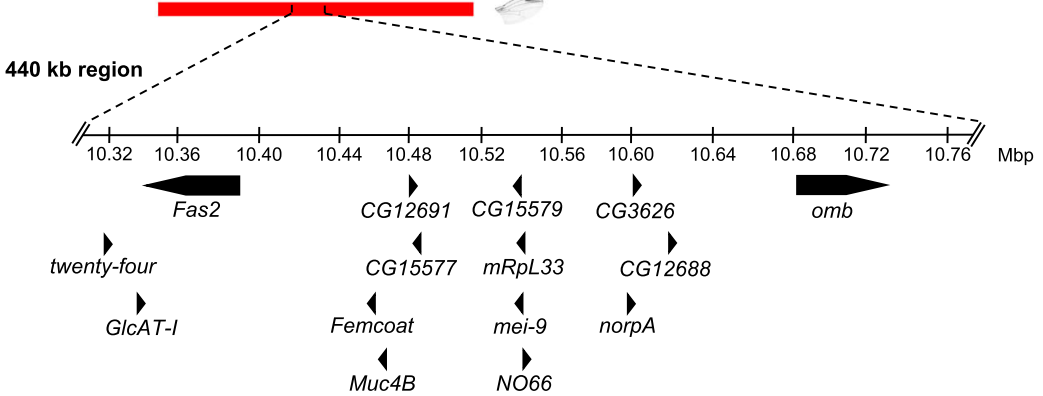

Figure 2. QTL analysis, effect plots, and in situ hybridization for wing pigmentation divergence. (A) Wing spots vary in size and shape in $D$. elegans and $D$. gunungcola backcross recombinants. Wing spots were traced (pink) and quantified relative to proxies for total wing area (length $x$ width) using ImageJ software (see Methods). (B) Wing spot QTL map for the $D$. elegans (red) and $D$. gunungcola (blue) backcross. LOD (logarithm of the odds) is indicated on the $y$-axis. The $x$-axis represents the physical map of Muller Elements X, B, C, D, $\mathrm{E}$, and $\mathrm{F}$ based on the $D$. elegans assembled genome (see Methods). $D$. elegans and $D$. gunungcola have six chromosomes (Yeh et al. 2006; Yeh and True 2014) that correspond to $D$. melanogaster chromosomes as follows: $X=X, B=2 L, C=2 R, D=3 L, E=3 R$, and $F=4$. Individual SNP markers are indicated with black tick marks along the $x$-axis. Horizontal red and blue lines mark $P=0.01$ for the $D$. elegans and $D$. gunungcola backcross, respectively. (C) Effect plots for the $X$ chromosome QTL peak from the $D$. elegans backcross (left) and $D$. gunungcola backcross (right). Gray triangles represent individual replicates. (D) The chromosome region from X: 8-13 Mbp for backcross recombinants containing $X$ chromosome breakpoints immediately flanking the wing spot QTL peak were aligned to compare the effects of each on wing pigmentation. Regions in red represent $D$. elegans linked loci, and regions in blue represent $D$. gunungcola linked loci. Recombinants possessing $D$. elegans loci to the left of $\sim 10.32 \mathrm{Mbp}$ are spotless, whereas recombinants possessing $D$. elegans loci to the right of $\sim 10.74 \mathrm{Mbp}$ possess dark wing spots. (E) Two recombinants define the wing spot locus to a $\sim 440 \mathrm{Kbp}$ region containing 15 candidate genes. omb is a wing pigmentation candidate gene given evidence from prior work (see Results and Discussion). (F) In situ hybridization of $D$. elegans and $D$. gunungcola pupal wings probed for omb mRNA (purple) at $30 \mathrm{~h}$ after pupal formation (APF) (see Fig. $\mathbf{S 8}$ for additional replicates).

defining each "Wing spot size" were traced and the polygon area quantified in $\mathrm{mm}^{2}$ using the "Measure" function. "Wing spot size" varied between 0 (spotless) and $0.15 \mathrm{~mm}^{2}$ between recombinant individuals. Raw data for Figure 1D are available in File S3, and raw data for Figure 2 are deposited on Dryad (https://doi.org/10.5061/dryad.gb5mkkwm5).

\section{LIBRARY PREPARATION AND SEQUENCING}

We estimated chromosome ancestry "genotypes" for 724 D. elegans backcross progeny and 241 D. gunungcola backcross progeny with a single MSG (Andolfatto et al. 2011) library using
965 barcoded adaptors following methods described in Cande et al. (2012). In brief, to extract gDNA from all male backcross individuals, single flies were placed into individual wells of 96well (Corning, cat\# 3879) plates containing a single steel grinding bead in each well (Qiagen, cat\# 69989). Eleven plates in total were prepared for 965 individual gDNA extractions. gDNA was isolated and purified using the solid tissue extraction procedure from a Quick-DNA 96 Kit (Zymo, cat\# D3012) and a paint shaker to homogenize tissue. gDNA was tagmented using a hyperactive version of Tn5 transposase charged with annealed adaptor oligos following the methods described in Picelli et al. (2014). Unique 
barcoded adaptor sequences were ligated to each sample of tagmented gDNA with 14 cycles of PCR using OneTaq 2x Master Mix (NEB, cat\# M0482S), and all samples were pooled into a single multiplexed sequencing library. Agencourt AMPure XP beads (Beckman Coulter, cat\# A63881) were used to size select $\sim 150-800 \mathrm{bp}$ fragments and eluted in $35 \mu \mathrm{L}$ of molecular grade water (Corning, cat\# MT46000CI). The library was quantified by qPCR and sequenced in a single lane of Illumina HiSeq by the Janelia Quantitative Genomics Team.

In addition to generating the backcross sequencing library, both D. elegans $H K$ and D. gunungcola $S K$ parental species were sequenced at $20 \times$ coverage using an Illumina MiSeq Reagent Kit (version 3, 600 cycle PE). In brief, gDNA was extracted using a Quick-DNA Microprep Kit (Zymo, cat\# D4074) from 10 pooled females for each species and quantified on a Qubit 2.0 (Invitrogen). These samples were sent to the University of Michigan DNA Sequencing Core to prepare 300 bp paired-end libraries, which were quantified by $\mathrm{qPCR}$ and sequenced in a single lane of Illumina MiSeq.

\section{GENOME ASSEMBLY}

In brief, Illumina reads from all 965 backcross recombinants were used to perform MSG on the Baylor College of Medicine D. elegans genome assembly (accession number: GCA_000224195.2). Using custom scripts in R and Python (https://github.com/masseyj/elegans), the recombination fraction between the Baylor and MSG contigs was calculated and plotted to manually tabulate joins and splits between newly assembled contigs. These new contigs were then used to assemble approximately chromosome length scaffolds in D. elegans (accession number: WVIB00000000) and partially assembled scaffolds in D. gunungcola (accession number: WTSR00000000).

\section{MARKER GENERATION WITH MSG}

Following methods described previously (Andolfatto et al. 2011; Cande et al. 2012), we used the MSG software pipeline (https: //github.com/JaneliaSciComp/msg/tree/master/instructions) to perform data parsing and chromosome ancestry estimation to generate markers for QTL analysis. In brief, using data from the Illumina backcross sequencing library (see File $S 4$ for the number of reads per individual), we mapped reads to the assembled $D$. elegans and D. gunungcola parental genomes to estimate chromosome ancestry for each backcross individual. We generated 3425 and 3121 markers for the D. elegans and D. gunungcola backcrosses, respectively, for QTL analysis (markers, phenotypes, and procedures for QTL mapping are deposited on Dryad [https://doi. org/10.5061/dryad.gb5mkkwm5]). PDFs of chromosomal breakpoints for each recombinant are available here: https://deepblue. lib.umich.edu/data/concern/data_sets/j098zb17n?locale=en.

\section{QTL ANALYSIS}

QTL analysis was performed using R/qtl (Broman et al. 2003; Broman and Sen 2009) in $\mathrm{R}$ for Mac version 3.3.3 ( $\mathrm{R}$ Core Team 2018) in a MacOS environment. Ancestry data for both backcross directions were imported into R/qtl using a custom script (https://github.com/dstern/read_cross_msg), which directly imports the conditional probability estimates produced by the Hidden Markov Model (HMM) of MSG (Andolfatto et al. 2011). We performed genome scans with a single QTL model using the "scanone" function of R/qtl and Haley-Knott regression (Haley and Knott 1992) for "Wing spot size" and "Maximum wing display angle." Note, for "Wing spot size," 68 and 42 recombinants from the D. elegans and D. gunungcola backcross populations, respectively, were excluded from the QTL mapping because their wings were too damaged to quantify spot variation. Similarly, for "Maximum wing display angle," 314 and 94 recombinants from the D. elegans and D. gunungcola backcross populations, respectively, were excluded from the QTL mapping because these males did not perform any courtship behavior during the assay. Significance of QTL peaks at $\alpha=0.01$ was determined by performing 1000 permutations of the data. Effect sizes for each QTL peak were individually estimated by comparing the mean "Wing spot size" or "Maximum wing display angle" between individuals that inherited either D. elegans or D. gunungcola alleles at each QTL peak position (markers, phenotypes, and procedures for QTL mapping are deposited on Dryad [https://doi.org/10.5061/dryad.gb5mkkwm5]).

Because we detected multiple QTL peaks on separate chromosomes for "Maximum wing display angle," we tested for the presence of epistatic interactions using two methods: First, we performed two- and three-way ANOVAs comparing the effect of each QTL peak in multiple QTL peak genetic backgrounds and found no evidence of an interaction. For two-way ANOVAs, we tested for any statistically significant interactions for max wing display angles between two different QTL peaks in the D. elegans backcross. For three-way ANOVAs, we tested for any statistically significant interactions for max wing display angles between three different QTL peaks in the D. gunungcola backcross. Second, we performed genome-wide pairwise tests using the "scantwo" function of R/qtl and Haley-Knott regression to test for non-additive interactions across all markers; LOD significance thresholds at $\alpha=0.05,0.01$, and 0.001 were determined by performing 1000 permutations of the data for each model (Fig. S2; Tables S1 and S2).

\section{ANNOTATING THE WING SPOT QTL INTERVAL}

To annotate genes within the $\sim 440 \mathrm{Kbp}$ fine-mapped wing spot locus, we performed nucleotide BLAST (BLASTn) (Johnson et al. 2008) searches against the D. melanogaster genome (taxid: 7227) using $\sim 10-K b p$ windows of assembled D. elegans 
chromosomal regions spanning the wing spot QTL interval. Using the "GBrowse" tool on Flybase (Thurmond et al. 2018), we mapped regions of microsynteny to identify the orientation of each gene and exported the respective $D$. melanogaster coding region (CDS) FASTA sequences to align with the D. elegans $\mathrm{X}$ chromosome.

\section{IN SITU HYBRIDIZATION}

Fly genomic DNA (gDNA) was extracted from 10 homogenized D. elegans and D. gunungcola females using a Quick-DNA Microprep Kit (Zymo, cat\# D3021). The following forward and reverse primers were designed and synthesized by Integrated DNA Technologies (IDT) to PCR amplify $321 \mathrm{bp}$ DNA templates targeting exon 5 of the omb locus in $D$. elegans: 5'-GCTGAGGATCCATTCGCTAGATTTG-3' and 5'-GTTGTTGGAACTAGAGTTGTTGGTG-3', and D. $g u$ nungcola: 5'- GCTGAGGATCCATTCGCTAGATTTG-3' and 5'-GTTGTTGGAACTGGAGTTGTTGGTG-3'. Reverse primers were designed beginning with a T7 RNA polymerase binding sequence (TAATACGACTCACTATAG) to facilitate in vitro transcription. Raw PCR products were then used to generate digoxigenin-labeled RNA probes using a T7 RNA in vitro transcription kit (Promega/Life Technologies). RNA was ethanol precipitated and resuspended in water to analyze on a Nanodrop. Each probe was stored at $-20^{\circ} \mathrm{C}$ in $50 \%$ formamide before in situ hybridization.

All tissues underwent primary dissection in PBS (1X phosphate-buffered saline), fixed for $30 \mathrm{~min}$ in $4 \%$ PFA, washed three times in PBT (PBS $+1 \%$ Triton $\mathrm{X}-100)$, and underwent secondary dissection in PBT, and then washed two times in $\mathrm{MeOH}$ and two times in EtOH before being stored at $-20^{\circ} \mathrm{C}$. Male D. elegans and D. gunungcola $\mathrm{L} 3$ wing discs were dissected first to validate that our omb probes detected an mRNA expression pattern similar to D. melanogaster (Grimm and Pflugfelder 1996; Fig. S3). Next, pupal wings were dissected at 30 and $48 \mathrm{~h}$ after pupal formation (APF) to probe for $o m b$ mRNA. To prepare pupal wings, appropriately staged pupae underwent a primary dissection: they were cut in half along the anterior-posterior axis using Astra Platinum Double Edge Razor Blades, and fat body was washed out of the pupal casing using a pipette and PBS prior to fixation. After fixation, pupal wings underwent a secondary dissection to pull off the cuticle surrounding each wing and then washed using the procedure described above. Finally, in situ hybridization was carried out as previously described (Vincent et al. 2019). Briefly, we used an InsituPro VSi robot to rehydrate in $\mathrm{PBT}$, fix in $\mathrm{PBT}$ with $4 \% \mathrm{PFA}$, and prehybridize in hybridization buffer for $1 \mathrm{~h}$ at $65^{\circ} \mathrm{C}$. Samples were then incubated with probe for $16 \mathrm{~h}$ at $65^{\circ} \mathrm{C}$ before washing with hybridization buffer and PBT. Samples were blocked in PBT with $1 \%$ bovine serum albumin (PBT + BSA) for $2 \mathrm{~h}$. Samples were then incubated with anti-digoxigenin Fab fragments conjugated to alkaline phosphatase (Roche) diluted 1:6000 in PBT + BSA. After additional washes, color reactions were performed by incubating samples with NBT and BCIP (Promega) until purple stain could be detected under a dissecting microscope. Samples were mounted in glycerol on microscope slides coated with polyL-lysine and imaged at $10 \times$ magnification on a Leica DFC450C camera.

\section{GENERATING ADVANCED RECOMBINANT INTROGRESSIONS ON THE X CHROMOSOME}

To try to isolate the QTL effects for "Wing spot size" and "Maximum wing display angle" localized to the X chromosome according to the $D$. elegans backcross experiment, $F_{1}$ hybrid females were generated using the procedures described above. $\mathrm{F}_{1}$ hybrid females were then backcrossed to D. elegans males in $\sim 12$ replicate vials, and backcross males lacking wing spots were isolated to measure "Maximum wing display angles" during courtship as described above. This procedure was repeated for seven generations to generate BC3-BC9 backcross individuals: backcross females were backcrossed en masse (across $\sim 12$ vials) to D. elegans males, and BC3 backcross males lacking wing spots were isolated to measure "Maximum wing display angles" during courtship with D. elegans virgins (and so on to BC9). At each generation, an attempt was made to create stable introgression lines of advanced recombinant males lacking wing spots, but all failed to produce offspring, suggesting that $D$. gunungcola X-linked loci might also contain hybrid sterility factors. After seven generations of backcrossing, gDNA from all backcross males lacking wing spots was extracted and sequenced for MSG as described above. Backcross males lacking wing spots from BC4-BC9 were homozygous for D. elegans genomic regions across all autosomes but varied for the amount of $D$. gunungcola genome regions on the $\mathrm{X}$ chromosome.

\section{INTROGRESSION OF BLACK BODY COLOR ALLELES FROM D. GUNUNGCOLA INTO D. ELEGANS}

In the D. gunungcola backcross, QTL mapping for wing spot size revealed QTL peaks linked to Muller Element $\mathrm{C}$ and $\mathrm{E}$ when spotless recombinants were excluded from the analysis (Fig. S4; Table S3). The Muller Element E QTL peak is located near the ebony gene, which appears to contribute to variation in body color between D. elegans and D. gunungcola (unpubl. data). We therefore reasoned that introgressing dark body color from $D$. gunungcola into D. elegans would introgress the Muller Element E QTL peak underlying wing spot size differences. After six generations of backcrossing dark brown female recombinants with $D$. elegans males, we crossed dark brown male and female recombinants together to create black offspring homozygous for 
the introgressed region. We then performed MSG on a single, dark black introgression line and found that it was homozygous for $\sim 1.5 \mathrm{Mb}$ of D. gunungcola alleles linked near the Muller Element E QTL peak (Fig. S4A,C).

\section{OBSERVING AND COLLECTING WILD D. GUNUNGCOLA AND D. ELEGANS IN INDONESIA}

Throughout early July 2018, D. elegans and D. gunungcola were recorded performing courtship in East Java, Indonesia on Ipomoea sp. and Brugmansia sp. flowers using Canon VIXIA HF R500 camcorders mounted to Manfrotto (MKCOMPACTACNBK) aluminum tripods. Both species were observed in sympatry on flowers near Coban Rondo Waterfall in Batu, Batu City, East Java, Indonesia (-7.884985, 112.477311). After video recording courtship, males and females were captured using a mouth pipette and gently aspirated into glass vials containing standard fly media (glucose, corn meal, yeast extract, and agar). Isofemale lines of D. gunungcola from Bumiaji District (Batu City, East Java Province, Indonesia) were established in the laboratory on standard fly media at $24^{\circ} \mathrm{C}$ temperature. We quantified (see Methods-QUANTIFICATION OF WING DISPLAY BEHAVIOR), to the best of our knowledge, the first recorded observations of D. gunungcola wing displays on flowers in the field and in the laboratory (Fig. S11; Videos 8, 9, and 11). Across $\sim 10$ independent observations of courting D. gunungcola pairs at Bumiaji District and Coban Rondo, all males performed a type of wing display despite completely lacking wing spots. To confirm species identification of D. gunungcola and D. elegans from the field sites mentioned above, we dissected and imaged male genitalia and compared with the laboratory strains (D. gunungcola $S K$ and D. elegans $H K$ ) used in this study and described previously (Sultana et al. 1999; Kopp and True 2002) (Fig. S9). The distal paramere (also called the pregonite; Rice et al. 2019) was especially diagnostic of species identity (Fig. S9). We also performed low-coverage sequencing of the new D. gunungcola strains' genomes from Coban Rondo (see Methods-LIBRARY PREPARATION AND SEQUENCING) and aligned coding sequences from the omb locus with the $D$. gunungcola SK lab strain (Fig. S10). A nonsynonymous coding change that distinguished the laboratory D. gunungcola $S K$ strain from $D$. elegans $H K$ also distinguished the new D. gunungcola Coban Rondo strain from D. elegans HK, matching the $D$. gunungcola SK sequence (Fig. S10).

\section{STATISTICS}

Statistical tests were performed in $\mathrm{R}$ for Mac version 3.3.3 (R Core Team 2018) using Student's $t$-test (two-tailed) to test for statistically significant effects of pairwise comparisons of continuous data with normally distributed error terms. For tests comparing more than two groups, ANOVAs were performed with post hoc Tukey HSD for pairwise comparisons adjusted for multiple comparisons. See "QTL analysis" methods for statistical tests used during QTL mapping.

\section{Results and Discussion X-LINKED SEQUENCE DIVERGENCE CONTRIBUTED TO WING SPOT AND WING DISPLAY DIVERGENCE}

Drosophila elegans males perform elaborate wing display dances (Video 1) in front of females during courtship, displaying the presence of darkly pigmented wing spots (Fig. 1B), whereas its sibling species, D. gunungcola, lost wing spots (Yeh et al. 2006; Prud'homme et al. 2006) and wing displays (Fig. 1B; Video 2). Despite these differences in sexual traits, D. elegans and $D$. gunungcola can mate and form viable $\mathrm{F}_{1}$ hybrids in the lab (Yeh et al. 2006; Yeh and True 2014). Sequence divergence on the $X$ chromosome has previously been implicated in the divergence of wing spots and wing display behavior (Yeh et al. 2006; Yeh and True 2014). To confirm this effect of the X-chromosome, we quantified variation in wing spot size and wing display behavior in $F_{1}$ hybrid males from reciprocal crosses between D. elegans and D. gunungcola. These $\mathrm{F}_{1}$ hybrids inherited their X chromosome from either D. elegans or D. gunungcola (whichever species was their mother) and autosomes from both species. Consistent with prior work, $\mathrm{F}_{1}$ hybrid males inheriting the $\mathrm{X}$ chromosome from $D$. elegans mothers $\left(\mathrm{F}_{1} \mathrm{E}\right)$ possessed wing spots, whereas $\mathrm{F}_{1}$ hybrid males inheriting the $\mathrm{X}$ chromosome from D. gunungcola mothers $\left(\mathrm{F}_{1} \mathrm{G}\right)$ did not (Fig. 1C,D). These wing spots of $F_{1} E$ males were smaller, however, than the wing spots seen in D. elegans (Fig. 1D; Student's $t$-test, $P=$ 0.02). Differences in wing display behavior were also apparent between $F_{1} E$ (Video 3) and $F_{1} G$ hybrids (Video 4), which is also consistent with prior work (Yeh et al. 2006; Yeh and True 2014). More specifically, we found that although both $F_{1}$ hybrids performed wing displays during courtship, $\mathrm{F}_{1} \mathrm{E}$ hybrids tended to open their wings more widely than $F_{1} G$ hybrids during display performance (Fig. 1C). We quantified variation in this wing display trait between $F_{1}$ hybrids by measuring the maximum bilateral wing display angles (Fig. 1C) during courtship (see Methods). We found that $F_{1} E$ hybrids performed wing displays comparable to D. elegans males (Fig. 1E; post hoc Tukey HSD, $P=0.6$ ), whereas $\mathrm{F}_{1} \mathrm{G}$ males showed, on average, lower display angles (Fig. 1E; post hoc Tukey HSD, $P=7.1 \times 10^{-5}$ ). Together these data confirm that divergence of one or more loci on the $\mathrm{X}$ chromosome contributes to divergence in wing spot size and wing display behavior between D. elegans and D. gunungcola. 
Table 1. QTLs detected for wing spot size and maximum wing display angle divergence.

\begin{tabular}{llllll}
\hline Trait & Backcross & Chromosome & QTL interval (bp) $^{\text {a }}$ & QTL peak (bp) $^{\text {LOD }}$ \\
\hline Wing spot size & D. elegans & X & $10,297,836-10,744,020$ & $10,304,581$ & 220 \\
Max wing display angle & D. elegans & X & $8,729,737-15,691,924$ & $9,006,035$ & 18.9 \\
Max wing display angle & D. elegans & B & $5,773,911-13,325,000$ & $9,001,485$ & 4.66 \\
Wing spot size & D. gunungcola & X & $10,474,499-11,584,862$ & $11,223,359$ & 38.9 \\
Max wing display angle & D. gunungcola & X & $16,885,658-25,539,528$ & $24,196,217$ & 4.23 \\
Max wing display angle & D. gunungcola & B & $7,078,659-12,180,268$ & $10,093,006$ & 6.28 \\
Max wing display angle & D. gunungcola & E & $3,813,413-11,535,144$ & $9,604,970$ & 7.59 \\
\hline
\end{tabular}

a LOD drop 1.5 support interval.

\section{EVOLUTION OF AT LEAST THREE LOCI CONTRIBUTE TO WING SPOT DIVERGENCE}

To identify the location of X-linked (as well as autosomal) loci contributing to divergence in wing spot size, we quantified wing spot size variation in 656 recombinant males produced by backcrossing $\mathrm{F}_{1}$ hybrid females to $D$. elegans males and 199 recombinant males produced by backcrossing $\mathrm{F}_{1}$ hybrid females to D. gunungcola males. These backcross males showed a range of wing spot sizes (Fig. 2A). Using MSG (Andolfatto et al. 2011), we inferred the allele most likely inherited from the $F_{1}$ mother (D. elegans or D. gunungcola) for each genomic position in each recombinant. We then performed QTL mapping for wing spot size and identified a single, highly significant QTL peak on the X chromosome (Fig. 2B and Table 1). In both backcross directions, variation linked to this wing spot QTL peak explained almost all of the difference in wing spot size between D. elegans and D. gunungcola (Fig. 2C). Repeating the QTL mapping after excluding recombinant individuals lacking wing spots, however, allowed us to identify additional QTLs of smaller effect on Muller Elements $\mathrm{C}$ (chromosome 2R in D. melanogaster) and $\mathrm{E}$ (chromosome 3R in D. melanogaster) in the D. gunungcola (but not D. elegans) backcross population (Fig. S4A; Table S3). Observing these QTL only in the D. gunungcola backcross populations suggests that they are caused by recessive $D$. gunungcola alleles, which were never homozygous in the D. elegans backcross population. Introgressing the QTL region on Muller Element E from $D$. gunungcola into D. elegans through five generations of backcrossing (Fig. S4C) reduced the size of wing spots (Fig. S4D,E). This region includes the ebony gene, which has previously been shown to be able to inhibit the development of dark pigments in D. melanogaster (Wittkopp et al. 2002b). Crossing this introgression line to D. elegans masked most of the reduction in spot size (Fig. S4D,E), consistent with the D. gunungcola QTL allele being recessive to the $D$. elegans allele. Taken together, these data indicate that the majority of wing spot divergence between D. elegans and D. gunungcola maps to a single, large-effect QTL on the $\mathrm{X}$ chromosome, but that wing spot size is also influenced by loci on Muller Elements $\mathrm{C}$ and $\mathrm{E}$.

\section{A 440-kb LOCUS BEHAVES LIKE A GENETIC SWITCH FOR WING SPOTS}

To further refine the X-linked QTL, we more closely examined the genotypes and phenotypes of recombinants with inferred crossover positions immediately flanking the wing spot QTL peak (Figs. 2D and S5). Doing so allowed us to identify a $\sim 440$-kb region containing a QTL that acts like a genetic switch controlling the presence or absence of the wing spot (Figs. 2D and S5). This region includes 15 genes (Fig. 2E) and notably excludes the $\mathrm{X}$-linked pigmentation gene, yellow, which has previously been suggested to contribute to wing spot development and evolution through changes in a spot-specific cis-regulatory element (Wittkopp et al. 2002a; Gompel et al. 2005; Prud'homme et al. 2006; Yeh et al. 2006; Arnoult et al. 2013; Yeh and True 2014; Fig. S6). One of these 15 genes is optomotor-blind (omb) (Fig. 2E), which encodes a T-box-containing transcription factor (Pflugfelder et al. 1992a; Pflugfelder et al. 1992b) that has previously been implicated in pigmentation patterning (Thompson 1959; Kopp and Duncan 1997), pigmentation evolution (Brisson et al. 2004), and distal wing patterning (Grim and Pflugfelder 1996). In D. melanogaster, gain- and loss-of-function omb alleles cause expansion and contraction of abdominal pigmentation bands, respectively (Kopp and Duncan 1997), and variation in abdominal pigmentation patterning in Drosophila polymorpha is strongly associated with polymorphisms at the omb locus (Brisson et al. 2004).

Although we identified two nonsynonymous protein coding changes between D. elegans and D. gunungcola (File S5), omb is required for the development of many structures throughout the body (Pflugfelder 2009); we, therefore, reasoned that genetic divergence in $o m b$ would be more likely to affect its expression than its protein function (Stern and Orgogozo 2008). To look for differences in omb expression between D. elegans and $D$. gunungcola that might affect wing spot development, we used in situ hybridization to detect $o m b$ mRNA in the developing wing of both species (Fig. 2F). In D. melanogaster, omb is expressed in a broad stripe that overlaps the wing pouch region in larval L3 wing discs (Grimm and Pflugfelder 1996). omb expression in the 
A

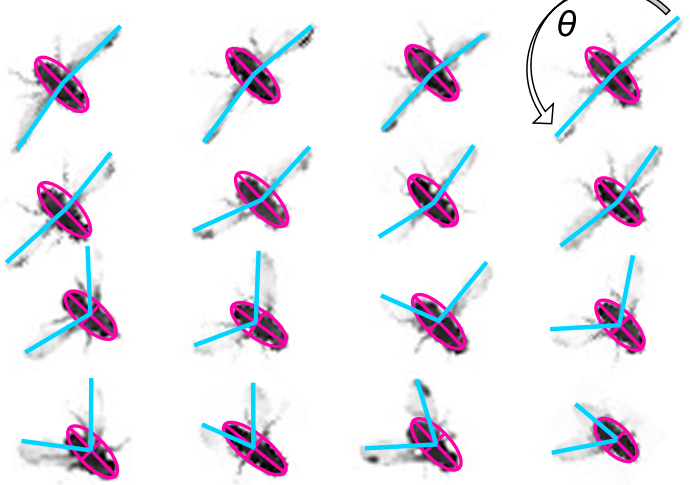

B

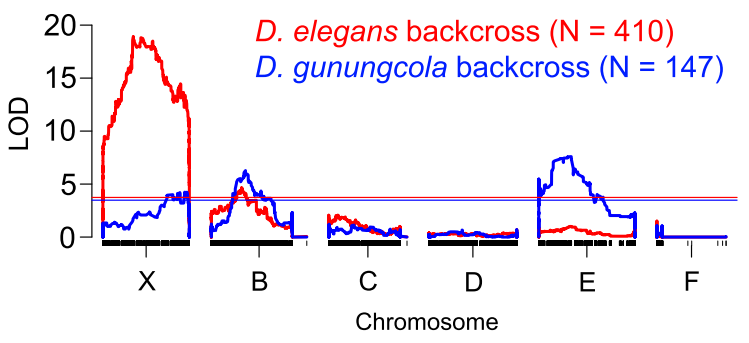

D. gunungcola backcross

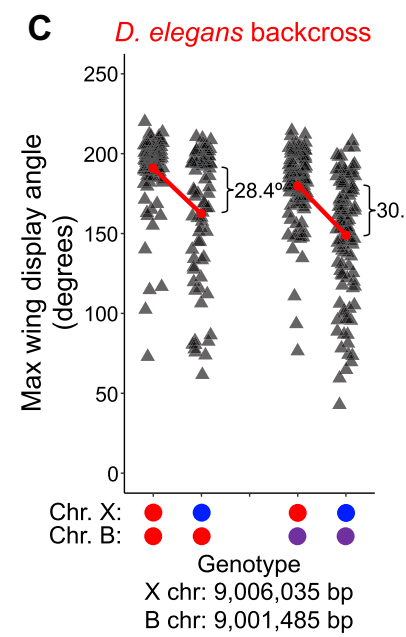

B chr: $9,001,485$ bp

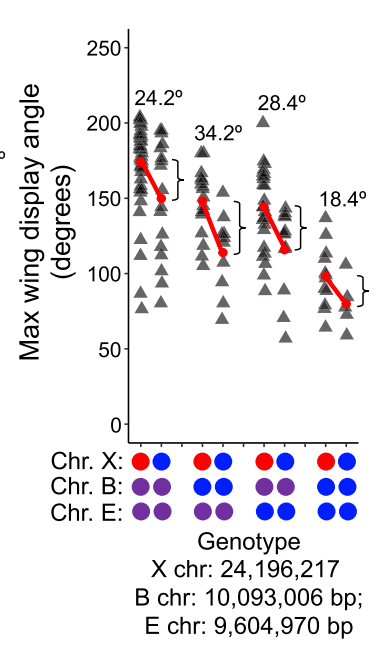

\begin{tabular}{c} 
Genotype Key \\
$\bullet D$. elegans $\bullet D$. gunungcola $\bullet$ Het. \\
\hline
\end{tabular}

Figure 3. QTL analysis and effect plots for wing display divergence. (A) Maximum wing display angles varied in $D$. elegans and $D$. gunungcola backcross recombinants. Maximum wing display angles were quantified by measuring the angle between each wing tip using ImageJ software (see Methods). (B) Maximum wing display QTL map for the $D$. elegans (red) and $D$. gunungcola (blue) backcross. LOD is indicated on the $y$-axis. Individual SNP markers are indicated with black tick marks along the $x$-axis. Horizontal red and blue lines mark $P=0.01$ for the $D$. elegans and $D$. gunungcola backcross, respectively. (C) Effect plots for the $\mathrm{X}$ chromosome and Muller Element B QTL peaks from the $D$. elegans backcross (left) and for the X, Muller Element B, and E QTL peaks from the D. gunungcola backcross (right). No epistatic interactions were detected wing pouch is required for distal wing development, as demonstrated by $D$. melanogaster omb hypomorphs that show disrupted distal wing tip development in adults (Grimm and Pflugfelder 1996). We hypothesized, therefore, that differences in D. elegans and D. gunungcola omb expression patterning during pupal wing development might prefigure changes in wing spot pigmentation observed in adult males, similar to the changes in wingless expression shown to prefigure wing spots in Drosophila guttifera (Werner et al. 2010). Consistent with the expression of omblacZ in pupal wings of D. melanogaster (Álamo Rodrı́guez et al. 2004), we detected $o m b$ mRNA in the wing hinge and distal wing tip $30 \mathrm{~h}$ APF in D. elegans and D. gunungcola (Fig. 2F). We were unable to identify any consistent differences in the omb expression patterns between D. elegans and D. gunungcola, although it is possible that we may not have detected subtle differences in expression patterns. In addition, it is possible that the changes in $o m b$ protein sequence contribute to differences in wing spot patterning, or that other genes in the minimal mapped interval are the true cause of the difference in wing spot patterning.

\section{EVOLUTION AT MULTIPLE LOCI CONTRIBUTED TO WING DISPLAY DIVERGENCE}

To identify loci contributing to divergence in wing display behavior, we quantified variation in maximum wing display angles (see Methods) in 410 D. elegans and 147 D. gunungcola backcross recombinant males, again observing a range of phenotypes (Fig. 3A). We identified multiple significant QTL contributing to variation in wing display (Fig. 3B; Table 1). In the D. elegans backcross, we mapped a QTL on the $\mathrm{X}$ chromosome that overlaps with the wing spot QTL (Fig. 3B; Table 1). We also mapped a QTL on Muller Element B (chromosome $2 \mathrm{~L}$ in D. melanogaster) (Fig. 3B; Table 1). In the D. gunungcola backcross, we mapped QTLs on the X chromosome as well as Muller Elements B and E (Fig. 3B; Table 1). The observation of a peak on Muller Element E only in the D. gunungcola backcross suggests the presence of alleles affecting wing display behavior that are recessive and/or interact epistatically with divergent sites elsewhere in the genome.

To test for epistatic interactions contributing to wing display divergence, we performed a two-dimensional genome scan to search for non-additive interactions across all markers in both backcross directions and found no significant interactions

between QTLs (see Methods) (two-way ANOVA: $F_{1,402}=0.146 ; P=$ 0.70 for the $D$. elegans backcross; three-way ANOVA: $F_{1,137}=0.050$ (X:B), 0.034 (X:E), 1.75 (B:E), and 0.799 (X:B:E); $P=0.82$ (X:B), 0.86 (X:E), 0.19 (B:E), and 0.37 (X:B:E) for the $D$. gunungcola backcross). Gray triangles represent individual replicates. 
(Fig. S2; Tables S1 and S2). We also tested for evidence of non-additive interactions among the wing display QTL peaks themselves by performing two- and three-way ANOVAs in the $D$. elegans and D. gunungcola backcrosses, respectively, and found no evidence of significant interactions between loci (Fig. 3C). Instead, each wing display QTL peak appears to behave approximately additively, with $D$. gunungcola alleles contributing to lower maximum wing display angles (Fig. 3C). Surprisingly, the effect of the X-linked QTL on wing display angle in the $D$. $g u$ nungcola backcross in multiple genetic backgrounds was similar to the estimated effect size of the X-linked QTL in the D. elegans backcross (compare panels in Fig. 3C) despite the much lower LOD score of the X-linked QTL in the D. gunungcola backcross population (Fig. 3B; Table 1). We suggest that although the detected QTL in the D. gunungcola backcross appear to interact additively with each other, undetected QTL elsewhere in the genome are likely masking the X-effect in the D. gunungcola backcross map. Although the purpose of the two-dimensional genome scan (Fig. S2; Tables S1 and S2) was to detect these effects, our sample size is likely too small to identify small-effect epistatic interactions.

\section{MALES LACKING WING SPOTS PERFORM NORMAL WING DISPLAYS}

Although it remains unclear which gene evolved to cause the majority of wing spot divergence, fine-mapping the locus controlling the presence or absence of the wing spot allowed us to test whether the locus that turns off wing spots in D. gunungcola also affects wing display behavior. To perform this test, we introgressed $D$. gunungcola alleles causing a loss of the wing spot into D. elegans by repeated backcrossing (see Methods). We recovered three introgression lines lacking wing spots and found that all three lines had inherited the $\sim 440-\mathrm{kb}$ region observed in mapping experiments to act like a genetic switch controlling wing spot development (Fig. 4A,B), independently confirming the causal role of the switch region in wing spot divergence. We noticed, however, that several advanced recombinants developed a wing spot "shadow" (Fig. 4B), possibly due to the effects of other D. elegans alleles affecting wing spot development. We next asked whether the spotless advanced recombinants performed wing displays with lower wing display angles than D. elegans males. Surprisingly, we found that all advanced recombinants inheriting the $D$. gunungcola allele eliminating the wing spot performed wing displays indistinguishable from $D$. elegans males during courtship (Fig. 4B,C; Videos 5-7). Thus, the loci controlling the wing spot and courtship behavior are genetically separable.

The repeated co-evolution of male-specific wing spots and wing display behavior in multiple species (Kopp and True 2002) combined with the presence of overlapping QTL for these traits
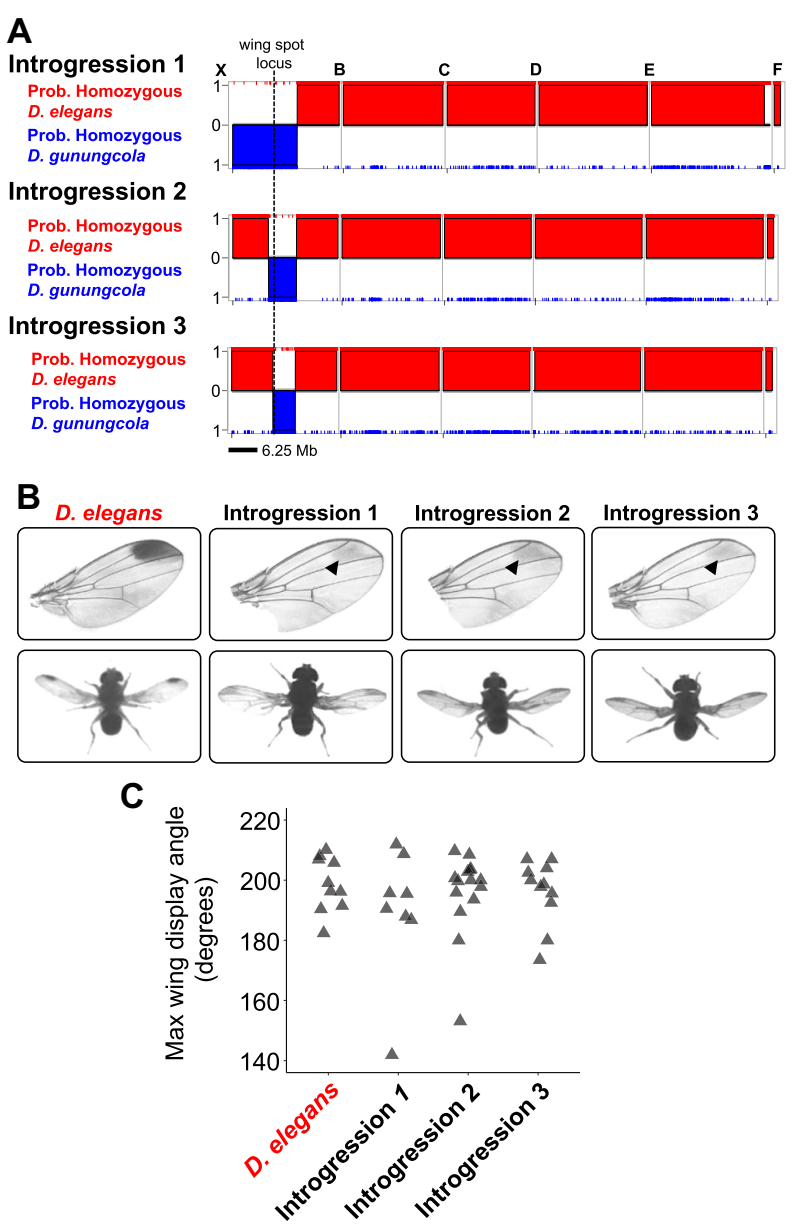

Figure 4. Drosophila elegans males possessing the $D$. gunungcola wing spot locus perform normal wing displays. (A) Multiplexed Shotgun Genotyping (MSG) (Andolfatto et al. 2011) was used to estimate genome-wide ancestry assignments for three introgression lines generated by repeatedly backcrossing the $D$. gunungcola wing spot QTL region into a $D$. elegans genetic background (see Methods). The posterior probability that a region is homozygous for $D$. elegans (red) or $D$. gunungcola (blue) ancestry is plotted along the $y$-axis. The dotted line marks the location of the fine-mapped wing spot region (Fig. 2D,E; Table 1). (B) None of the introgressions possessed dark wing spots (although a light wing spot "shadow" is visible). (B and C) Flies from all introgression lines performed max wing display angles indistinguishable from $D$. elegans males (one-way ANOVA: $F_{3,42}=0.449$; $P=0.72$ ). Gray triangles represent individual replicates.

on the X chromosome (Yeh et al. 2006; Yeh and True 2014; and this study) suggested that a single pleiotropic gene might be contributing to the evolution of both traits. The finding that D. elegans introgression lines lacking a wing spot performed a normal wing display argues against this hypothesis and indicates instead that these two traits arose independently between this species pair. To further investigate how these divergent traits might have evolved, we recorded courtship behavior in a wild 
population of D. gunungcola in Indonesia; to the best of our knowledge, all prior studies of D. gunungcola pigmentation and courtship used the one previously available lab strain (Sultana et al. 1999). Surprisingly, we found that all D. gunungcola males observed in the wild population lacked wing spots (Fig. S7) but performed wing displays (Fig. S11; Videos 8 and 9), confirming that these are genetically distinct traits. The wing displays performed by these flies appeared to show a lower maximum wing extension angle than D. elegans (Fig. S11), similar to the wing display behavior seen in $\mathrm{F}_{1}$ hybrids between D. elegans and D. gunungcola with D. gunungcola mothers (Fig. 1C; Video 4). Analysis of new lab strains founded by flies captured from this D. gunungcola population showed similar male courtship behavior in the lab as observed on flowers (Fig. S11; Video 11).

It remains unknown whether the absence of wing display behavior in the D. gunungcola $S K$ lab strain seen since Yeh et al. (2006) (collected originally from Sumatra in 1999) is either (1) segregating within and/or among wild populations of D. gunungcola or (2) limited to the SK strain and might have been lost by chance or adaptation to the lab environment. Observing that flies from the newly isolated strain of D. gunungcola displayed a similar wing display behavior in the field and after being reared in the lab (Fig. S11) does, however, argue that the absence of wing display behavior in the SK line is unlikely due to phenotypic plasticity caused by the lab setting. Additional sampling of natural D. gunungcola populations from throughout its species range (or at least resampling of the population from which the SK lab line was derived) is needed to distinguish between these possibilities. What we can say at this time, however, is that the new strain of D. gunungcola performed a similar wing display in the field and in the lab, with the angle of the wing display appearing to be consistently less than the wing display angle measured for D. elegans (Fig. S11). Because we mapped QTL explaining variation in the wing display angle (Fig. 3) rather than the presence or absence of wing display, some QTL identified here might also contribute to variation in wing display angle segregating within wild populations. We therefore conclude that although the absence of wing spots appears fixed in $D . g u$ nungcola, the absence of wing display behavior does not. These observations suggest that the loss of male-specific wing spots predates the loss of male wing display behavior in this species.

\section{Conclusions}

Male-specific wing spots and wing display behavior have coevolved in Drosophila multiple times (Kopp and True 2002). By studying the genetic basis of these divergent traits between $D$. elegans and D. gunungcola, we showed that the changes in wing spot and wing display were not caused by changes in a single, pleiotropic gene despite overlapping QTL (Yeh et al. 2006; Yeh and True 2014). Rather, we found that distinct loci contribute to divergence in each of these traits, with the genetic architecture of divergent wing behavior being more complex than that of the divergent wing spot pigmentation. Both traits were affected by divergent gene(s) located on the $\mathrm{X}$ chromosome that are in physical linkage, however, causing alleles of these distinct loci to be co-inherited. This linkage might have facilitated the coordinated evolution of these traits.

The specific genes contributing to divergence in wing spot and wing display remain unknown, but optomotor-blind is a strong candidate for the X-linked gene contributing to the loss of the wing spot. Introgression lines and additional sampling of $D$. gunungcola from a wild population also showed that the loss of wing spots and wing display are not inexorably linked: in both cases, males lacking wing spots still performed a wing display behavior. Coordinated evolution of morphological and behavioral traits such as these is often observed in animal species, but it is often unclear which change evolved first. In this case at least, it seems that the divergence of morphology preceded the divergence of behavior.

\section{AUTHOR CONTRIBUTIONS}

JHM was associated with conceptualization, data curation, formal analysis, funding acquisition, validation, investigation, visualization, methodology, and writing (original draft, and review and editing). GRR was associated with formal analysis, validation, investigation, methodology, and writing (review and editing). ASF was associated with investigation, methodology, writing (review and editing). Chi-Yang Chen was associated with investigation and methodology. SDY was associated with funding acquisition, investigation, and methodology. DLS was associated with supervision, funding acquisition, conceptualization, data curation, formal analysis, investigation, visualization, writing (original draft and review and editing), and project administration PJW was associated supervision, funding acquisition, conceptualization, data curation, formal analysis, investigation, visualization, writing (original draft and review and editing), and project administration.

\section{ACKNOWLEDGMENTS}

We thank members of the Wittkopp, Stern, and Rebeiz labs for helpful discussions. For fly strains, we thank J. True (Stony Brook University). For guidance throughout the in situ hybridization work, we thank M. Rebeiz (University of Pittsburgh). For arranging the Material Transfer Agreement for D. gunungcola and D. elegans, we thank N. Kurniawan (Department of Biology, University of Brawijaya, Indonesia); for hosting us in Indonesia, we thank K. Wicaksono (Faculty of Agriculture, University of Brawijaya, Indonesia); for assistance with field collections, we thank H. Tarno (Faculty of Agriculture, University of Brawijaya, Indonesia). Funding was provided by University of Michigan, Department of Ecology and Evolutionary Biology, Peter Olaus Okkelberg Research Award, National Institutes of Health (NIH) training grant T32GM007544, and Howard Hughes Medical Institute Janelia Graduate Research Fellowship to JHM; NIH R01 GM089736 and 1R35GM118073 to PJW. 


\section{DATA ARCHIVING}

All supporting data can be accessed at University of Michigan Deep Blue (https://deepblue.lib.umich.edu/data/concern/data_sets/j098zb17n? locale=en) and Dryad (https://doi.org/10.5061/dryad.gb5mkkwm5).

\section{LITERATURE CITED}

Andolfatto, P., D. Davison, D. Erezyilmaz, T. T. Hu, J. Mast, T. Sunayama-Morita, and D. L. \&Stern. 2011. Multiplexed shotgun genotyping for rapid and efficientgenetic mapping. Genome Res. 21: 610-617.

Álamo Rodrıǵuez, D., J. T. Felix, and F. J. Diáz-Benjumea. 2004. The role of the T-box geneoptomotor-blind in patterning the Drosophila wing. Dev. Biol. 268:481-492.

Arnoult, L., K. F. Su, D. Manoel, C. Minervino, J. Magriña, N. Gompel, and B. Prud'homme. 2013. Emergence and diversification of fly pigmentation through evolution of a generegulatory module. Science 339:14231426.

Bock, I. R., and M. R. Wheeler. 1972. The Drosophila melanogaster species group. Univ. Tex. Publs. 7213:1-102.

Brisson, J. A., A. R. Templeton, and I. Duncan. 2004. Population genetics of thedevelopmental gene optomotor-blind (omb) in Drosophila polymorpha: evidencefor a role in abdominal pigmentation variation. Genetics 168:1999-2010.

Broman, K. W., and S. Sen. 2009. A guide to QTL mapping with R/qtl. Vol. 46. Springer, New York.

Broman, K. W., H. Wu, S. Sen, and G. A. Churchill. 2003. R/qtl: QTL mapping in experimental crosses. Bioinformatics 19:889-890.

Cande, J., P. Andolfatto, B. Prud'homme, D. L. Stern, and N. Gompel. 2012. Evolution of multiple additive loci caused divergence between Drosophila yakuba and D.santomea in wing rowing during male courtship. PLoS ONE 7:e43888.

Chang, S. H., S. Jobling, K. Brennan, and D. J. Headon. 2009. Enhanced Edar signalling has pleiotropic effects on craniofacial and cutaneous glands. PLOS ONE 4. e7591.

Charlesworth, D., and B. Charlesworth. 1976. Theoretical genetics of Batesian mimicry II. Evolution of supergenes. J. Theor. Biol. 55: 305-324.

Duveau, F., and M. A. Félix. 2012. Role of pleiotropy in the evolution of a cryptic developmental variation in Caenorhabditis elegans. PLoS Biol. 10. e1001230.

Endler, J. A. 1991. Variation in the appearance of guppy color patterns to guppies and their predators under different visual conditions. Vision Res. 31:587-608.

Endler, L., J. M. Gibert, V. Nolte, and C. Schlötterer. 2018. Pleiotropic effects of regulatory variation in tan result in correlation of two pigmentation traits in Drosophila melanogaster. Mol. Ecol. 27:3207-3218.

Gompel, N., B. Prud'homme, P. J. Wittkopp, V. A. Kassner, and S. B. Carroll. 2005. Chance caught on the wing: cis-regulatory evolution and the origin of pigment patterns in Drosophila. Nature 433:481-487.

Gray, S. M., and J. S. McKinnon. 2007. Linking color polymorphism maintenance and speciation. Trends Ecol. Evol. 22:71-79.

Grimm, S., and G. O. Pflugfelder. 1996. Control of the gene optomotorblind in Drosophila wing development by decapentaplegic and wingless. Science 271:1601-1604.

Haley, C. S., and S. A. Knott. 1992. A simple regression method for mapping quantitative trait loci in line crosses using flanking markers. Heredity 69:315-324.

Johnson, M., I. Zaretskaya, Y. Raytselis, Y. Merezhuk, S. McGinnis, and T. L. Madden. 2008. NCBI BLAST: a better web interface. Nucleic Acids Res. 36:W5-W9.
Kent, C. F., T. Daskalchuk, L. Cook, M. B. Sokolowski, and R. J. Greenspan. 2009. The Drosophila foraging gene mediates adult plasticity and geneenvironment interactions in behaviour, metabolites, and gene expression in response to food deprivation. PLoS Genet. 5:e1000609.

Kirkpatrick, M., and N. Barton. 2006. Chromosome inversions, local adaptation and speciation. Genetics 173:419-434.

Kopp, A., and I. Duncan. 1997. Control of cell fate and polarity in the adult abdominal segments of Drosophila by optomotor-blind. Development 124:3715-3726.

Kopp, A., and J. R. True. 2002. Evolution of male sexual characters in the oriental Drosophila melanogaster species group. Evol. Dev. 4:278-291.

Kronforst, M. R., L. G. Young, D. D. Kapan, C. McNeely, R. J. O’Neill, and L. E. Gilbert. 2006. Linkage of butterfly mate preference and wing color preference cue at the genomic location of wingless. Proc. Natl. Acad. Sci. 103:6575-6580.

Küpper, C., M. Stocks, J. E. Risse, N. dos Remedios, L. L. Farrell, S. B. McRae, T. C. Morgan, N. Karlionova, P. Pinchuk, Y. I Verkuil, et al. 2016. A supergene determines highly divergent male reproductive morphs in the ruff. Nat. Genet. 48:79-83.

Lamichhaney, S., G. Fan, F. Widemo, U. Gunnarsson, D. S. Thalmann, M. P. Hoeppner, S. Kerje, U. Gustafson, C. Shi, H. Zhang, et al. 2016. Structural genomic changes underlie alternative reproductive strategies in the ruff (Philomachus pugnax). Nat. Genet. 48:84-88.

Lindholm, A., and F. Breden. 2002. Sex chromosomes and sexual selection in poeciliid fishes. Am. Nat. 160:S214-S224.

Loxton, R. G. 1979. On display behaviour and courtship in the praying mantis Ephestiasula amoena (Bolivar). Zool. J. Linnean Soc. 65:103-110.

McKinnon, J. S., and M. E. Pierotti. 2010. Colour polymorphism and correlated characters: genetic mechanisms and evolution. Mol. Ecol. 19:5101-5125.

McLean, C. Y., P. L. Reno, A. A. Pollen, A. I. Bassan, T. D. Capellini, C. Guenther, V. B. Indjeian, X. Lim, D. B. Menke, B. T. Schaar, et al. 2011. Human-specific loss of regulatory DNA and the evolution of humanspecific traits. Nature 471:216-219.

Merrill, R. M., P. Rastas, S. H. Martin, M. C. Melo, S. Barker, J. Davey, W. O. McMillan, and C. D. Jiggins. 2019. Genetic dissection of assortative mating behavior. PLoS Biol. 17:e2005902.

Nagy, O., I. Nuez, R. Savisaar, A. E. Peluffo, A. Yassin, M. Lang, D. L. Stern, D. R. Matute, J. R. David, and V. Courtier-Orgogozo. 2018. Correlated evolution of two copulatory organs via a single cis-regulatory nucleotide change. Curr. Biol. 28:3450-3457.

Pflugfelder, G. O. 2009. omb and circumstance. J. Neurogenet. 23:15-33.

Pflugfelder, G. O., H. Roth, and B. Poeck. 1992a. A homology domain shared between Drosophila optomotor-blind and mouse Brachyury is involved in DNA binding. Biochem. Biophys. Res. Comm. 186:918-925.

Pflugfelder, G. O., H. Roth, B. Poeck, S. Kersher, H. Schwarz, B. Jonschker, and M. Heisenberg. 1992b. The lethal (1) optomotor-blind gene of Drosophila melanogaster is a major organizer of optic lobe development: isolation and characterization of the gene. Proc. Nat. Acad. Sci. 89:1199-1203.

Picelli, S., Å. K. Björklund, B. Reinius, S. Sagasser, G. Winberg, and R. Sandberg. 2014. Tn5 transposase and tagmentation procedures for massively scaled sequencing projects. Genome Res. 24:2033-2040.

Prud'homme, B., N. Gompel, A. Rokas, V. A. Kassner, T. M. Williams, S. D. Yeh, J. R. True, and S. B. Carroll. 2006. Repeated morphological evolution through cis-regulatory changes in a pleiotropic gene. Nature 440:1050-1053.

R Core Team. 2018. R: a language and environment for statistical computing. Available via http://www.r-project.org/

Rice, G., J. R. David, Y. Kamimura, J. P. Masly, A. P. Mcgregor, O. Nagy, S. Noselli, M. D. S. Nunes, P. O’Grady, E. Sánchez-Herrero, et al. 
2019. A standardized nomenclature and atlas of the male terminalia of Drosophila melanogaster. Fly 13:51-64.

Simon, J. C., and M. H. Dickinson. 2010. A new chamber for studying the behavior of Drosophila. PLoS ONE 5:e8793.

Sinervo, B., D. B. Miles, W. A. Frankino, M. Klukowski, and D. F. DeNardo. 2000. Testosterone, endurance, and Darwinian fitness: natural and sexual selection on the physiological bases of alternative male behaviors in side-blotched lizards. Horm. Behav. 38:222-233.

Stern, D. L., and V. Orgogozo. 2008. The loci of evolution: how predictable is genetic evolution?Evolution 62:2155-2177.

Sultana, F., M. T. Kimura, and M. J. Toda. 1999. Anthophilic Drosophila of the elegans species-subgroup from Indonesia, with description of a new species (Diptera: Drosophilidae). Entomol. Sci. 2:121-126.

Thomas, J. W., M. Cáceres, J. J. Lowman, C. B. Morehouse, M. E. Short, E. L. Baldwin, D. L. Maney, and C. L. Martin. 2008. The chromosomal polymorphism linked to variation in social behavior in the white-throated sparrow (Zonotrichia albicollis) is a complex rearrangement and suppressor of recombination. Genetics 179:1455-1468.

Thompson, P. E. 1959. Periodical new mutants. Drosophila Inf Serv. 33:99.

Thurmond, J., J. L. Goodman, V. B. Strelets, H. Attrill, L. S. Gramates, S. J. Marygold, B. B. Matthews, G. Millburn, G. Antonazzo, V. Trovisco, et al. 2018. FlyBase 2.0: the next generation. Nucleic Acids Res. 47:D759-D765.

Vincent, B. J., G. R. Rice, G. M. Wong, W. J. Glassford, K. I. Downs, J. L. Shastay, K. Charles-Obi, M. Natarajan, M. Gogol, J. Zeitlinger, et al.
2019. An atlas of transcription factors expressed in male pupal terminalia of Drosophila melanogaster. G3 9:3961-3972.

Werner, T., S. Koshikawa, T. M. Williams, and S. B. Carroll. 2010. Generation of a novel wing colour pattern by the Wingless morphogen. Nature 464:1143-1148.

Wirtz, R. A., and H. G. Semey. 1982. The Drosophila kitchen - equipment, media preparation, and supplies. Drosoph. Inf. Serv. 58:176-180.

White, T. E., J. Zeil, and D. J. Kemp. 2015. Signal design and courtship presentation coincide for highly biased delivery of an iridescent butterfly mating signal. Evolution 69:14-25.

Wittkopp, P. J., K. Vaccaro, and S. B. Carroll. 2002a. Evolution of yellow gene regulation and pigmentation in Drosophila. Curr. Biol. 12: 1547-1556.

Wittkopp, P. J., J. R. True, and S. B. Carroll. 2002b. Reciprocal functions of the Drosophila yellow and ebony proteins in the development and evolution of pigment patterns. Development 129:1849-1858.

Yeh, S. D., and J. R. True. 2014. The genetic architecture of coordinately evolving male wing pigmentation and courtship behavior in Drosophila elegans and Drosophila gunungcola. G3 4:2079-2093.

Yeh, S. D., S. R. Liou, and J. R. True. 2006. Genetics of divergence in male wing pigmentation and courtship behavior between Drosophila elegans and D. gunungcola. Heredity 96:383-395.

Associate Editor: P. Schmidt Handling Editor: D. W. Hall

\section{Supporting Information}

Additional supporting information may be found online in the Supporting Information section at the end of the article.

Supplementary Table S2. Results of two-QTL scan for max wing display angle in D. elegans backcross

Supplementary Table S3. Results of two-QTL scan for max wing display angle in D. gunungcola backcross

Supplementary Table S1. QTLs detected for wing spot size, excluding spotless individuals

Video 1 D. elegans HK wing display behavior

Video 2 D. gunungcola SK courtship and copulation

Video $3 \mathrm{~F}_{1} \mathrm{E}$ wing display behavior

Video $4 \mathrm{~F}_{1} \mathrm{G}$ wing display behavior

Video 5 Introgression 1 wing display behavior

Video 6 Introgression 2 wing display behavior

Video 7 Introgression 3 wing display behavior

Video 8 D. gunungcola wing display behavior at Coban Rondo Waterfall in East Java, Indonesia (Version 1)

Video 9 D. gunungcola wing display behavior at Coban Rondo Waterfall in East Java, Indonesia (Version 2)

Video 10 D. elegans wing display behavior in Tumpang, Indonesia

Video 11 D. gunungcola (Batu City, Indonesia) wing display behavior in the laboratory 